\title{
Growth in systems of vesicles and membranes
}

\author{
A.M. Somoza ${ }^{a, c}$, U. Marini Bettolo Marconi ${ }^{b}$ and P. Tarazona ${ }^{c}$ \\ ${ }^{a}$ Instituto de Ciencia de Materiales, Consejo Superior de Investigaciones Científicas, \\ Madrid-28049, Spain \\ ${ }^{b}$ Dipartimento di Matematica e Fisica, Universita degli Studi di Camerino, via Madonna delle \\ Carceri, I-62032 Camerino, Italy \\ ${ }^{c}$ Departamento de Física de la Materia Condensada and Instituto Nicolas Cabrera, Universidad \\ Autónoma de Madrid, Madrid E-28049, Spain
}

(July 18, 2018)

\begin{abstract}
We present a theoretical study for the intermediate stages of the growth of membranes and vesicles in supersaturated solutions of amphiphilic molecules. The problem presents important differences with the growth of droplets in the classical theory of Lifshitz-Slyozov-Wagner, because the aggregates are extensive only in two dimensions, but still grow in a three dimensional bath. The balance between curvature and edge energy favours the nucleation of small planar membranes, but as they grow beyond a critical size they close themselves to form vesicles. We obtain a system of coupled equations describing the growth of planar membranes and vesicles, which is solved numerically for different initial conditions. Finally, the range of parameters relevant in experimental situations is discussed.
\end{abstract}

\section{INTRODUCTION}

Solutions of amphiphilic molecules in water may form a large variety of molecular aggregates, as a result of the asymmetric interaction with water of the hydrophilic heads and the hydrophobic tails of these molecules [1 5]. These aggregates range from micelles, which may be considered as large molecular clusters, to continuous structures of the amphiphile with regular or irregular structures such as sponge phases. In between, one finds structures like membranes and vesicles which are macroscopic in two dimensions, whereas they are only a few molecular sizes thick. From a thermodynamic point of view one can regard membranes as two dimensional phases in coexistence with a diluted bulk solution of water and amphiphile molecules, but contrary to the case of adsorbed layers on solid substrate, the membrane is not restricted to lie on fixed positions and orientations. The membrane is free to explore the full three dimensional space, and in the case of "fluid membranes", the lack of rigid molecular order within the membrane surface allows the bending of the membranes, with low energetic cost as "curvature energy" [6]. The extraordinary properties of membranes as self-assembling, self-sealing, insulating and flexible structures are used in biological systems 
to form the basis of cellular membranes, plasts and mitocondrial structures, the insulating material for neurons, etc. In the last decade, the study of membranes and other amphiphilic aggregates has attracted the attention of experimental and theoretical physicists, chemists, physical-chemists, biologists $[7-10$.

In the present paper we discuss the dynamics of formation of these aggregates from a supersaturated bulk solution, by extending to the peculiarities of these systems the methods developed for "regular" three dimensional phases. We focus our interest on amphiphilic systems forming two types of isolated bilayer aggregates: membranes and vesicles. During the formation of these macroscopic aggregates we may distinguish two different stages:

(i) A nucleation process, during which the microscopic aggregates form.

(ii) A growth process, when these seeds reach mesoscopic sizes or dissolve into the bulk solution, by following a nearly deterministic dynamics which transforms the initial population of the microscopic clusters into a distribution of sizes for the aggregates and depletes the amphiphile concentration in bulk solution to the equilibrium value.

In an infinite system at zero temperature, the growth process would never end. Alternatively, we should consider a third regime when the super-saturation is so small that the gradients in the chemical potential are affected by fluctuations.

Experimentally, these aggregates can be obtained with the help of techniques designed to accelerate the nucleation process. Among these are the ultrasound technique and the use of solid substrates (heterogeneous nucleation), which preferentially adsorb the amphiphilic molecules. On the other hand, the spontaneous formation from an equilibrium super-saturated solution is of interest. In fact, it has been suggested [12 that, due to the possibility of spontaneous formation, amphiphilic systems forming vesicles played a crucial role in the beginning of life, providing for the compartmentation of the early metabolic and self-reproducing molecular machinery.

In this work we address the growth process, in which the aggregates formed in the early stage compete with each other to grow, incorporating the amphiphilic molecules from the super-saturated solution. We try to answer the questions about how the typical distribution of sizes evolves and how it depends on the distribution in the early stage. Our model needs as input the initial distribution of sizes as well as the initial super-saturation of the system. This data could be obtained from nucleation theory, but the study of the different mechanisms of nucleation requires molecular models of these complex systems, which are still in an early stage of development.

\section{THE MODEL}

Let us consider the possible equilibrium shapes for bilayer aggregates. For a fixed area, $A$, a symmetric bilayer adopts the shape that minimizes the effective Helfrich Hamiltonian [6]:

$$
\mathcal{H}=\int d A\left\{\frac{1}{2} \kappa\left(C_{1}+C_{2}\right)^{2}+\kappa_{G} C_{1} C_{2}\right\}+\lambda \oint d l
$$

where the first integral extends over the whole surface area and the other one extends along the boundary, where $\kappa$ and $\kappa_{G}$ are respectively the bending rigidity and the Gaussian 
curvature moduli, $\lambda$ is the line tension and $C_{1}, C_{2}$ are the two principal local curvatures of the surface. In the case of a flat circular membrane of radius $R, \mathcal{H} \propto R$, whereas for a spherical vesicle of radius $S, \mathcal{H}=4 \pi\left(2 \kappa+\kappa_{g}\right)$. Thus, small aggregates tend to assume a planar shape but larger aggregates will always prefer a closed shape. To locate the spontaneous shape transformation we employ a simple spherical cap model where the bilayer is restricted to adopt the shape of the curved surface of a sphere cut by a plane. For a fixed area the shape can be fully described by the radius, $R$, of the circle of intersection between the sphere and the plane,

$$
\mathcal{H}(A, R)=4 \pi \kappa_{s}\left(1-\frac{\pi R^{2}}{A}\right)+2 \pi \lambda R,
$$

where $\kappa_{s}=2 \kappa+\kappa_{G}$. A spherical vesicle corresponds to $R=0$ while a planar membrane is described by $R=(A / \pi)^{1 / 2}$. In Figure 1 we display the energy $\mathcal{H}$ versus $R$ for different values of $A$. The absolute minimum is always at an extremum: for $A<4 \pi\left(\kappa_{s} / \lambda\right)^{2}$ it corresponds to planar membranes while for $A>4 \pi\left(\kappa_{s} / \lambda\right)^{2}$ it corresponds to spherical vesicles. When the two configurations have the same energy $\left(A=4 \pi\left(\kappa_{s} / \lambda\right)^{2}\right)$, they are separated by an energy barrier $\Delta \mathcal{H}=3 \pi \kappa_{s}$, which decreases with increasing $A$ and finally disappears at $A_{c}=\pi\left(4 \kappa_{s} / \lambda\right)^{2}$, where all planar membranes become unstable and close into vesicles.

Such a simple model gives a first glimpse of the growth process: unless the nucleation mechanism is strongly biased to produce vesicles, the early population of aggregates consist of planar membranes; as some of these grow they become metastable with respect to the closed vesicles, but still separated from them by a barrier, until their area reaches the critical value $A_{c}$. At this stage the membranes become unstable and turn into closed vesicles of the same area, which can grow until the solution has been depleted down to the equilibrium concentration of amphiphiles.

We start constructing our model of growth for a system of both vesicles and membranes by making the following assumptions:

(i) The excess concentration (with respect to a dilute solution without aggregates) is so small that the interaction between aggregates can be neglected.

(ii) All aggregates are much larger than their typical thickness $(\xi \approx 40 \AA)$. This assumption is expected to hold at least in the long time regime.

(iii) The main mechanism for transport of amphiphiles is diffusion as expected when the water solution is at rest, so that all hydrodynamic effects can be neglected. In this sense it is implied that the growth process does not agitate the water solution.

(iv) We consider an infinite volume.

(v) Finally we assume that fluctuations can be neglected.

We shall later comment on some of these assumptions. Within this approximations the problem clearly resembles the classical Lifshitz-Slyozov-Wagner (LSW) theory for the growth of spherical domains [13, [1].

Within a quasi-equilibrium thermodynamic description the chemical potential, $\mu(\mathbf{r})$, changes smoothly with position. We consider an isolated growing aggregate of area $A$ and $N(A)$ molecules; its rate of growth will depend on the number of amphiphilic molecules that approach its surface:

$$
\frac{d N(A)}{d t}=-\oint \mathbf{j} \cdot d \mathbf{S}
$$


where the integral extends over a surface enclosing the aggregate and $\mathbf{j}(\mathbf{r})$ is the current of amphiphilic molecules induced by the gradients of chemical potential which is different in the bulk and at the surface of the aggregate:

$$
\mathbf{j}=-\alpha \nabla \mu(\mathbf{r})
$$

$\alpha$ being a kinetic coefficient. Assuming that the transport of amphiphiles does not change its local concentration,

$$
\nabla \cdot \mathbf{j}=0
$$

everywhere outside the border of the aggregate. This leads to a Poisson equation for $\mu(\mathbf{r})$, equivalent to an electrostatic potential with boundary conditions at infinity, $\mu_{\infty}$, and at the surface of the aggregate $\mu(A)$, which mimics an equipotential metallic boundary. Such a value, $\mu(A)$, depends on the particular properties of each aggregate.

\section{A. Membranes.}

Let us consider a system composed only of membranes and assume that none of them transforms into vesicles. The excess of grand potential energy of a planar circular membrane of radius $R$ is

$$
\Delta \Omega_{m}(R)=\pi \sigma R^{2}+2 \pi \lambda R
$$

where $\sigma$ is the surface tension of the membrane and $\lambda$ is the line tension associated with the boundaries. The equilibrium of a large membrane of arbitrary shape is controlled by the requirement $\sigma=0$, which at a given temperature is satisfied when the chemical potential $\mu$ assumes its equilibrium value $\mu_{o}$. In general, for a fixed chemical potential $\mu$, the surface tension does not vanish but is given by:

$$
\sigma(\mu)=-\Gamma\left(\mu-\mu_{0}\right)
$$

where $\Gamma$ is the adsorption per unit area in the membrane. Since we are considering systems with small supersaturations, within a linear approximation we take the values of $\lambda$ and $\Gamma$ evaluated at $\mu=\mu_{o}$. For $\mu>\mu_{0}$ eq. (6) has a maximum at

$$
R_{c}(\mu)=\frac{\lambda}{\Gamma\left(\mu-\mu_{0}\right)}
$$

In other words, membranes with $R>R_{c}(\mu)$ tend to adsorb particles and grow. This growth is much faster than the diffusion process described above (provided there are particles to be absorbed in the neighbourhood of the membrane), and it will stop only when the adsorption of particles has effectively changed the chemical potential in the proximity of the membrane. We thus obtain the boundary condition of the diffusion problem:

$$
\mu(R)=\mu_{0}+\frac{\lambda}{\Gamma R}
$$


An equivalent argument applies for shrinking membranes when $\mu<\mu_{0}$. Now it is possible to get the chemical potential field for an isolated membrane at an arbitrary distance $\mathbf{r}$ from its center. Neglecting the thickness of the membrane is equivalent to know the electrostatic potential created by a planar metallic disk [16]:

$$
\mu(\mathbf{r})=\mu_{\infty}+\frac{2\left(\mu(R)-\mu_{\infty}\right) R}{\pi r} \sum_{l=0}^{\infty} \frac{(-1)^{l}}{2 l+1}\left(\frac{R}{r}\right)^{2 l} P_{2 l}(\cos \theta)
$$

for $R<r$, where $\mathbf{r}=(r, \theta, \phi)$ in spherical coordinates. From eq. (雨) and (3), the rate of growth of an isolated membrane of radius $R$ is

$$
\frac{d R}{d t}=\frac{4 \alpha \lambda}{\pi \Gamma^{2}}\left(\frac{\Gamma \Delta}{\lambda}-\frac{1}{R}\right)
$$

where $\Delta=\mu_{\infty}-\mu_{0}$ is a measure of the supersaturation of the system.

We turn now to the study of an ensemble of isolated membranes by introducing a size distribution function, $f_{m}(R, t)$ which gives the number of membranes of radius $R$ per unit volume at time $t . f_{m}(R, t)$ verifies the continuity equation

$$
\frac{\partial f_{m}}{\partial t}+\frac{\partial}{\partial R}\left(f_{m} v_{R}\right)=0
$$

where $v_{R}=d R / d t$ given by eq. (11). Finally, a closed set of equations is obtained by imposing the conservation of the total amount of amphiphiles:

$$
\chi \Delta(t)+\int_{0}^{\infty} d R \pi R^{2} \Gamma f_{m}(R, t)=Q,
$$

where the first term represents the excess number of particles per unit volume that remain in solution ( $\chi$ is the bulk compressibility) and the integral gives the number of particles per unit volume that belong to the membranes. With initial conditions for $f_{m}(R, t)$ and $\Delta(t)$ the system of equations (11), (12) and (13) is fully determined. Following the ideas of the LSW theory it is straight-forward to get the asymptotic behaviour of the system (see for example [15]). We summarize the main results:

$$
\begin{gathered}
\Delta(t)=\left(\frac{\pi \lambda}{2 \alpha t}\right)^{1 / 2} \\
N(t)=\frac{Q \Gamma}{\alpha \lambda I t}, \\
\bar{R}(t)=R_{c}(t)=\left(\frac{2 \alpha \lambda t}{\pi \Gamma^{2}}\right)^{1 / 2}, \\
f_{m}(R, t)=\frac{N(t)}{R_{c}(t)} P\left(\frac{R}{R_{c}(t)}\right),
\end{gathered}
$$


where $N(t)$ is the total number of membranes per unit volume, $\bar{R}(t)$ is the mean radius and $P(x)$ and $I$ are

$$
\begin{gathered}
P(x)= \begin{cases}\frac{8 x}{(2-x)^{4}} \exp \left(-\frac{2 x}{2-x}\right) & 0 \leq x \leq 2 \\
0 & 2<x,\end{cases} \\
I=\int_{0}^{\infty} d x x^{2} P(x)=1.1094
\end{gathered}
$$

It is important to note that within the present model the growth rate $R \propto t^{1 / 2}$ with a growth exponent $n=1 / 2$ instead of $1 / 3$ as predicted by LSW. Such a difference is due to the two-dimensional nature of the aggregate, which allows for a faster growth.

Our asymptotic formulae are expected to be valid for

$$
\log ^{2}\left(\frac{4 \alpha \Delta_{0}^{2}}{\pi \lambda} t\right) \gg 1
$$

where $\Delta_{0}$ is the initial supersaturation of the system. Unfortunately this equation does not always hold because before reaching a long enough time some membranes can transform into vesicles. Thus, this restriction must hold before $R_{c}(t) \leq 2 R_{T} \approx 8 k_{s} / \lambda$. From eq. (16), eliminating $t$ in (20)

$$
\log ^{2}\left(\frac{8 \Gamma \Delta_{0}^{2} k_{s}}{\lambda^{4}}\right) \gg 1
$$

Taking rough estimates of the parameters shown in Table I we get 18 for the left-hand-side of eq. (reflog2). Thus, real systems hardly reach this universal distribution function.

\section{B. Vesicles.}

We now consider the case of a system formed only by spherical vesicles and no planar membranes. In the case of an isolated vesicle of radius $S$, the excess grand potential is:

$$
\Delta \Omega_{v}=-4 \pi \Gamma S^{2}\left(\mu-\mu_{0}\right)+4 \pi \kappa_{s}
$$

It is clear from this equation that all vesicles, independently of their size, will grow until the chemical potential in the surroundings is $\mu_{0}$. Thus, it is easy to get the three equations that define the problem:

$$
\begin{gathered}
\frac{d S}{d t}=\frac{\alpha \Delta}{2 \Gamma} \\
\frac{\partial f_{v}}{\partial t}+\frac{\partial}{\partial S}\left(f_{v} v_{S}\right)=0,
\end{gathered}
$$


and

$$
\chi \Delta(t)+\int_{0}^{\infty} d S 4 \pi S^{2} \Gamma f_{v}(S, t)=Q,
$$

This set of equations lends itself to an analytical solution in terms of the initial conditions. The main result is that the size distribution function does not change its shape,

$$
f_{v}(S, t)=f_{0}\left(S-\frac{\alpha}{2 \Gamma} \int_{0}^{t} d t^{\prime} \Delta\left(t^{\prime}\right)\right)
$$

where $f_{0}(S)$ represents the initial distribution of vesicles. In this case $\Delta(t)$ decays exponentially. The reason for this behaviour is clear from eq. (23), which indicates that all vesicles grow at the same rate independently of their size.

It is interesting to notice that eqs. (23-25) were obtained under the assumption that the slowest growth process is the diffusion of particles (diffusion limited growth). But, for a vesicle to grow, it is necessary to fill the interior with water, thus a certain amount of water needs to overcome an energy barrier when crossing the bilayer structure. Under some circumstances this can be the slowest process, and then eq. (23) should be modified. In this case the number of water molecules that cross the bilayer per unit time will be proportional

to the area of the vesicle. For small enough supersaturations (or large enough curvature constant) the shape of the vesicle will remain spherical, and the change in volume is :

$$
\frac{d V}{d t}=4 \pi S^{2} \frac{d S}{d t}=4 \pi S^{2} F(\Delta) \approx C S^{2} \Delta
$$

where $F(\Delta)$ is a function related to the properties of the barrier that verifies $F(0)=0$ and, thus, it is expected to show a linear behaviour for small supersaturations, $C$ being a constant. This equation is equivalent to eq. (23) except for a constant, thus the basic result, eq. (26) still holds except for the time scale.

\section{Membranes and Vesicles.}

When there are no more membranes closing themselves to form vesicles, the distribution function $f_{v}$ does not change in shape, it merely moves to larger sizes until all the excess particles in the solution are exhausted. But in order to relate the distribution function when the nucleation process finishes it is necessary to allow for the existence of both membranes and vesicles and permit the spontaneous transformation from one to the other. We study this case assuming that this transformation occurs at one particular radius, $R_{T}=4 \kappa_{s} / \lambda$ and $S_{T}=R_{T} / 2$, (estimated from the spherical cap model) and the time scale of the process is totally negligible compared with diffusion times. Now we have to consider size distribution functions for both membranes and vesicles.

$$
\begin{gathered}
\frac{\partial f_{m}}{\partial t}+\frac{\partial}{\partial R}\left(v_{R} f_{m}\right)=-\delta\left(R-R_{T}\right) I_{m} \\
\frac{\partial f_{v}}{\partial t}+\frac{\partial}{\partial S}\left(v_{S} f_{v}\right)=\delta\left(S-S_{T}\right) I_{m}
\end{gathered}
$$




$$
\chi \Delta(t)+\int_{0}^{\infty} d R \pi R^{2} \Gamma f_{m}(R, t)+\int_{0}^{\infty} d S 4 \pi S^{2} \Gamma f_{v}(S, t)=Q,
$$

where $I_{m}$ is the number of membranes per unit volume that transform into vesicles at time $t, I_{m}=\max \left(0, v_{R_{T}} f_{m}\left(R_{T}, t\right)\right.$.

We can change to dimensionless units:

$$
\begin{array}{ll}
R=x L, S=x L / 2, & L=\frac{\lambda \chi}{\Gamma Q}, \\
t=\tau T, & T=\frac{\pi \lambda \chi^{2}}{4 \alpha Q^{2}}, \\
\Delta=\delta D, & D=\frac{Q}{\chi}, \\
f_{m}=f_{v}=\varphi F, & F=\frac{\Gamma^{2} Q^{4}}{\pi \lambda^{3} \chi^{3}},
\end{array}
$$

where we have made use of the fact that there are no membranes for $R>R_{T}$ and there are no vesicles for $S<S_{T}$ to join both size distribution functions into one single function $\varphi(x, \tau)$ : for $x<x_{T} \equiv R_{T} / L$ it represents membranes of radius $R=x L$ and for $x>x_{T}$ vesicles of radius $S=x L / 2$. With these changes of variable the new equations read:

$$
\begin{array}{lll}
\frac{\partial \varphi}{\partial \tau}+\frac{\partial}{\partial x}\left(\left(\delta-\frac{1}{x}\right) \varphi\right)=0 & \text { for } & x<x_{T} \\
\frac{\partial \varphi}{\partial \tau}+\frac{\partial}{\partial x}\left(\frac{\pi}{4} \delta \varphi\right)=0 & \text { for } & x>x_{T},
\end{array}
$$

with a special boundary condition at $x=x_{T}$, and

$$
\delta(\tau)+\int_{0}^{\infty} d x x^{2} \varphi(x, \tau)=1
$$

These equations have been solved numerically. We have applied a standard upwind algorithm [17] discretizing both in time and in space with $\Delta \tau=0.02$ and $\Delta x=0.1$. The initial conditions were chosen having in mind the particular values of the parameters shown in Table I. We consider that initially we only have membranes and assume that the size distribution function is gaussian with mean $\bar{R}_{i}=10 \psi \approx 4 \times 10^{-8} \mathrm{~m}$ and a width $\sigma_{i}=$ $\bar{R}_{i} / 4=10^{-8} \mathrm{~m}$. Our study applies when the nucleation process is already completed. The energy barrier for the formation of new membranes must be much lower that $k_{B} T$; this means that $\Delta_{0} \ll \pi \lambda^{2} /\left(\Gamma k_{B} T\right) \approx 8 \times 10^{-21} \mathrm{~J} /$ molec. We have selected $\Delta_{0}=3 \times 10^{-23} \mathrm{~J} /$ molec which corresponds to a critical radius $R_{c}(0)=3 \bar{R}_{i} / 4$. The only parameter that remains to be fixed is the height of the initial distribution, or equivalently the value of $Q$. We arbitrarily consider that at the initial time $90 \%$ of the total excess of particles is already in a membrane whereas only the remaining $10 \%$ is solved in water, leading to $Q=8 \times 10^{16} \mathrm{molec} / \mathrm{m}^{3}$. The results of the calculation are shown in Figure 2. Instead of the size distribution function (whose total integral tends to zero in time) we plot $x^{2} \varphi(x, \tau)$ which is proportional to the probability of finding a molecule in an aggregate of size $x$ at time $\tau$, and its total integral tends to one. In the early stage the distribution function moves to larger sizes and spreads, decreasing the height of the peak. At $\tau \approx 1300$ some membranes start to transform into vesicles and the rate increases until $\tau \approx 3000$ when it starts to decrease. At $\tau \approx 5200$ the critical radius becomes larger than the transformation radius and, thus all membranes shrink. At that time the final distribution function for vesicles is known; it will just translate 
to larger sizes absorbing all remaining particles in the solution and in the membranes. In Figure 3 is shown the critical radius, $x_{c}$ versus $\tau$. For these initial conditions the behaviour is quite monotonous. I is almost linear from $\tau=500$ to $\tau=6000$. For larger times, when most of the membranes have disappeared we would reach the expected exponential behaviour.

A totally different behaviour can be obtained from different initial conditions. The results are shown in Figures 4 and 5. The only difference with respect to the previous case is that we have assumed that at the initial time only $5 \%$ (instead of 90\%) of the total excess of particles is forming the initial membranes. In the early time the critical radius is lower than the mean radius and there is a large amount of material to be absorbed; this permits a very fast growth of the membranes without broadening the distribution function and as soon as $\tau \approx 100$ there are already some vesicles formed (this early time growth process is not shown in the figure because it is covered by later data). This fast growth also makes the critical radius increase and, as soon as some membranes are formed, it crosses the peak of the distribution of membranes. As the distribution function is highly peaked this crossing suddenly makes most of the membranes shrink. For a period of time there is an equilibrium where the critical radius remains constant as the vesicles grow at the expense of the membranes. When the number of membranes is small the critical radius crosses the transformation value. The final distribution function of membranes is highly peaked as a result of the very fast early growth.

\section{CONCLUSIONS.}

We have presented a study of the growth processes for amphiphilic membranes. These are two dimensional aggregates in a three dimensional bath, with peculiar features: the surface vs. volume balance which controls the growth for droplets is changed to a line vs. surface balance for planar membranes, or it may be avoided by closed vesicles, without open edge, at the price of a size independent curvature energy. Our work here extends the classical LifshitzSlyozov-Wagner theory to consider this problem. We have shown that, contrary to the nucleation of droplets, there is no asymptotic limit in which the size distribution of aggregates becomes independent of the initial conditions. As shown by our numerical solutions of the coupled equations for planar membranes and spherical vesicles, the asymptotic form of the distribution function for vesicles changes dramatically with the initial configuration. Allowing for the large uncertainty in the experimental values of several parameters in the Table I, and for the overall complexity of the problem, our equations may provide a first guide to the systematic understanding of these processes.

It is important to make a comment regarding the absence of fluctuations in our treatment. The LSW theory neglects fluctuations because it is expected that in average the concentration will follow the gradients in chemical potential created by the existence of aggregates. But this assumption conflicts with some other limits taken in the theory in particular large times, i.e. large domains, and small supersaturations. In this case the gradients created by the growth process can be of the same order or lower than the gradients created by fluctuations in the concentration. That means that some aggregates could shrink even though their radius is larger than the critical radius and this effect can change the size

distribution function at late enough times. In particular for vesicles, this theory predicts a 
complete degeneracy in the equilibrium size distribution while fluctuations are expected to break this degeneracy imposing the the distribution function which maximizes entropy [18].

\section{ACKNOWLEDGMENTS}

This work was supported by the Dirección General de Investigación Científica y Técnica (Spain) under grants number PB91-0090 and PB95-0005. 


\section{REFERENCES}

[1] Lindman B Physics of Amphiphiles: Micelles, Vesicles and Microemulsions, Eds. V. Degiorgio and M. Corti, (Amsterdam:North-Holland) (1985).

[2] Seddon J M Biochimica et Biophysica Acta 10311 (1990).

[3] Eds. Gelbart W M, Roux D and Ben-Shaul A Micelles, Membranes, Microemulsions and Monolayers. (Berlin:Springer) (1994).

[4] Gompper G and Schick M Self-assembling amphiphilic systems in Phase Transitions and Critical Phenomena ed. C. Domb and J. Lebowitz, (London:Academic Press) (1994).

[5] Israelachvili J N Physics of Amphiphiles: Micelles, Vesicles and Microemulsions, Ed. V. Degiorgio and M. Corti, (Amsterdam:North-Holland) (1985).

[6] Helfrich W., Z. Naturforsch 28c, 693 (1973).

[7] Nelson D, Piran T and Weinberg S (eds.) Statistical Mechanics of Membranes and Surfaces (Singapore:World Scientific) (1989)

[8] Lipowski R., Nature 349, 475 (1991).

[9] Lipowski R., Richter D. and Kremer K. (eds.) The Structure and Conformation of Amphiphilic Membranes (Berlin:Springer) (1992).

[10] Gennis R. B. Biomembranes: Molecular Structure and Function (Berlin:Springer) (1989).

[11] Gunton J.D. and Droz M. Introduction to the Theory of Metastable and Unstable States (Berlin:Springer) (1983).

[12] J.D. Watson, N.H. Hopkins, J.W. Robert, J.A. Steitz and A.M. Weiner, Molecular Biology of the Gene Benjamin/Cummings (1989).

[13] I. M. Lifshitz and V. V. Slyozov, J. Phys. Chem. Solids, 19, 35 (1961).

[14] C. Wagner, Z. Elektrochem. 65, 581 (1961).

[15] I. M. Lifshitz and L. P. Pitaevskii, Physical Kinetics, Landau and Lifshitz Course of Theoretical Physics, vol. 10, Pergamon (1981).

[16] See for example G. Arfken Mathematical Methods for Physicists, Third edition (1985), Academic page 661.

[17] W. H. Press, S. A. Teukolsky, W. T. Vetterling and B. P. Flannery; Numerical Recipes in Fortran, (second edition), Cambridge University Press, (1992).

[18] D. C. Morse and S. T. Milner; Europhys. Lett., 26, 565 (1994). 


\section{TABLES}

TABLE I. Orders of magnitude of some parameters used in the text. $R_{T}$ has been estimated from the spherical cap model, and see text Section II.C for the estimation of $\Delta_{0}$.

$k_{B} T 4 \times 10^{-21} \mathrm{~J}$

$\rho_{v} \quad 10^{18} \quad$ molec $/ \mathrm{m}^{3}$

$\chi \quad 2.4 \times 10^{38}$ molec $^{2} / \mathrm{J}$

$\begin{array}{lll}k_{s} & 10^{-18} \quad J\end{array}$

$\lambda \quad 10^{-11} \mathrm{~J} / \mathrm{m}$

Г $10^{19} \quad$ molec $/ m^{2}$

$R_{T} \quad 2 \times 10^{-7} \quad m$

$\Delta_{0} \quad 3 \times 10^{-23} \mathrm{~T} /$ molec 


\section{FIGURES}

FIG. 1. Curvature energy of a membrane as a function of its shape for a spherical cap model (see text). $\mathcal{H}(A, R)$ is the curvature energy (in units of $\kappa_{s}$ ) for a membrane of area, $A$, and edge circle of radius, $R$ (in dimensionless units). $u=R(\pi / A)^{1 / 2}$, where $u=0$ corresponds to spherical vesicles and $u=1$ corresponds to planar membranes. The area for the spontaneous shape transition is $A_{c}=\pi\left(4 \kappa_{s} / \lambda\right)^{2}$

FIG. 2. Time evolution of the size distribution function in dimensionless units, see eq. (31), for initial conditions where $90 \%$ of the excess particles particles are forming membranes (see text for details). $x=x_{T}=66.7$ is the radius for the spontaneous shape transition from membrane to vesicle. The region $x<x_{T}$ refers to planar membranes and $x>x_{T}$ to spherical vesicles.

FIG. 3. Critical Radius versus time in dimensionless units, see eq. (31), for initial conditions where $90 \%$ of the excess particles particles are forming membranes (see text for details). The dotted line corresponds to $x=x_{T}$.

FIG. 4. Time evolution of the size distribution function in dimensionless units, see eq. (31), for initial conditions where $5 \%$ of the excess particles particles are forming membranes (see text for details). $x=x_{T}=66.7$ is the size of spontaneous shape transition from membrane to vesicle. The region $x<x_{T}$ refers to planar membranes and $x>x_{T}$ to spherical vesicles.

FIG. 5. Critical Radius versus time in dimensionless units, see eq. (31), for initial conditions where $5 \%$ of the excess particles particles are forming membranes (see text for details). The dotted line corresponds to $x=x_{T}$. 


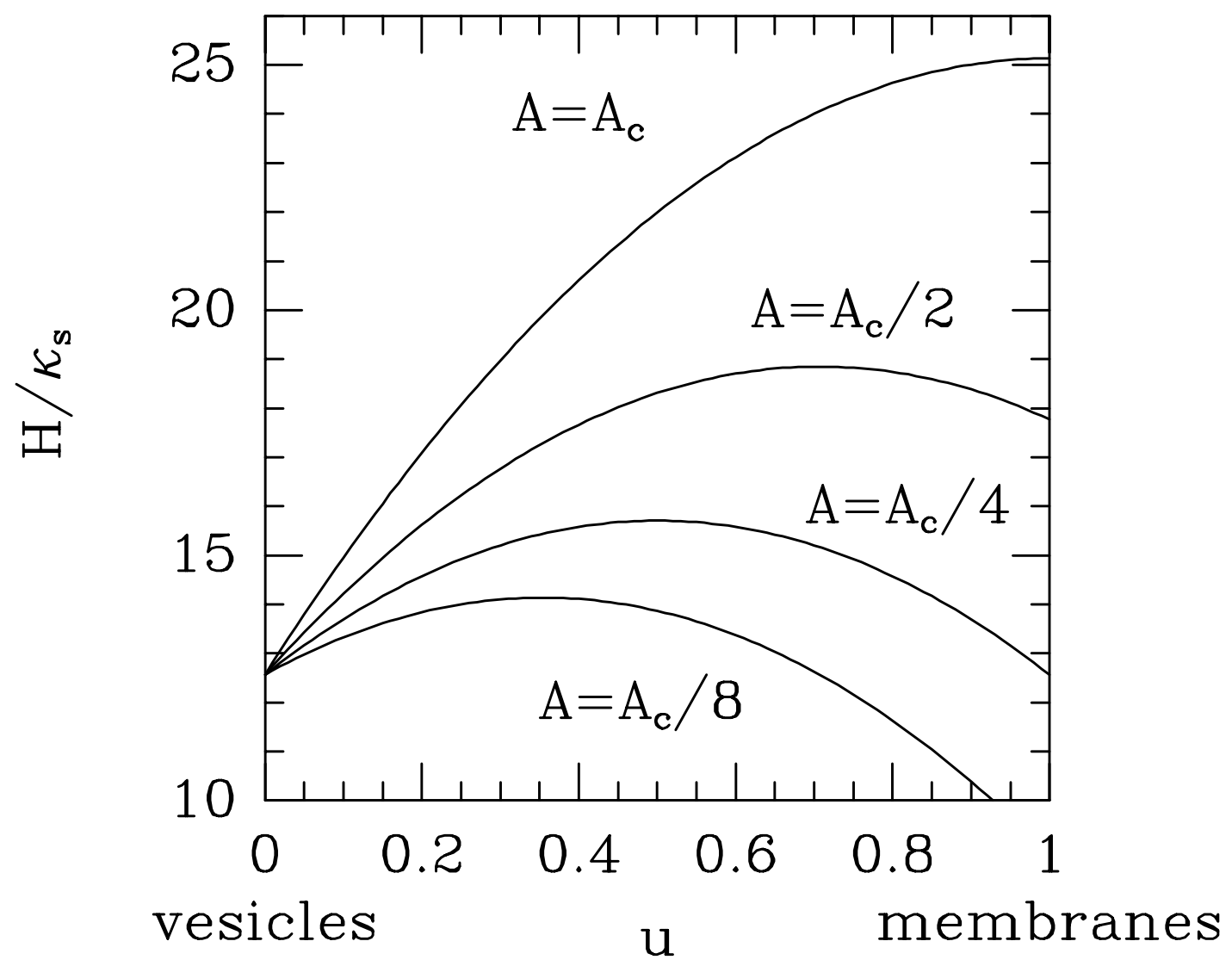




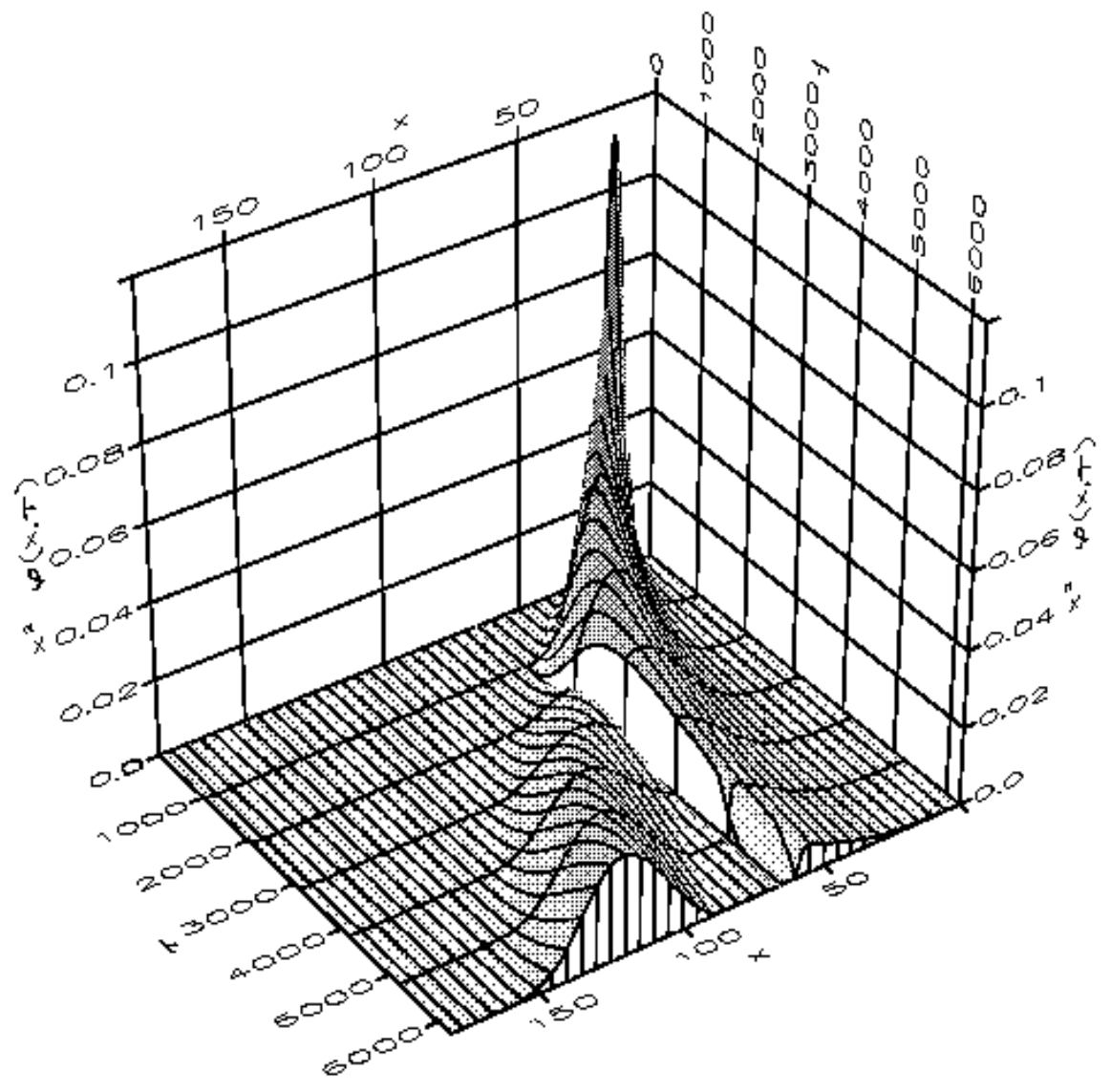




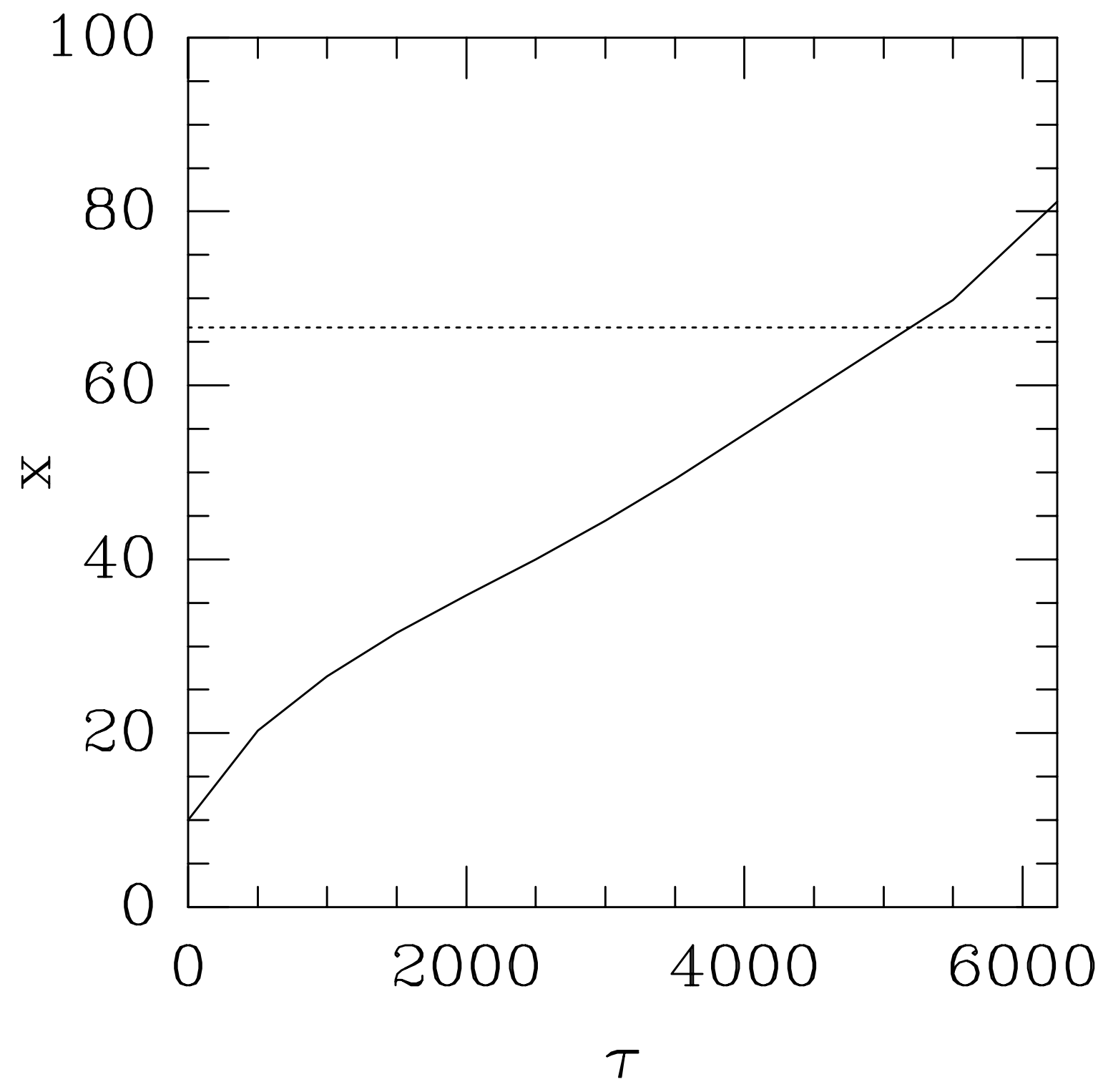




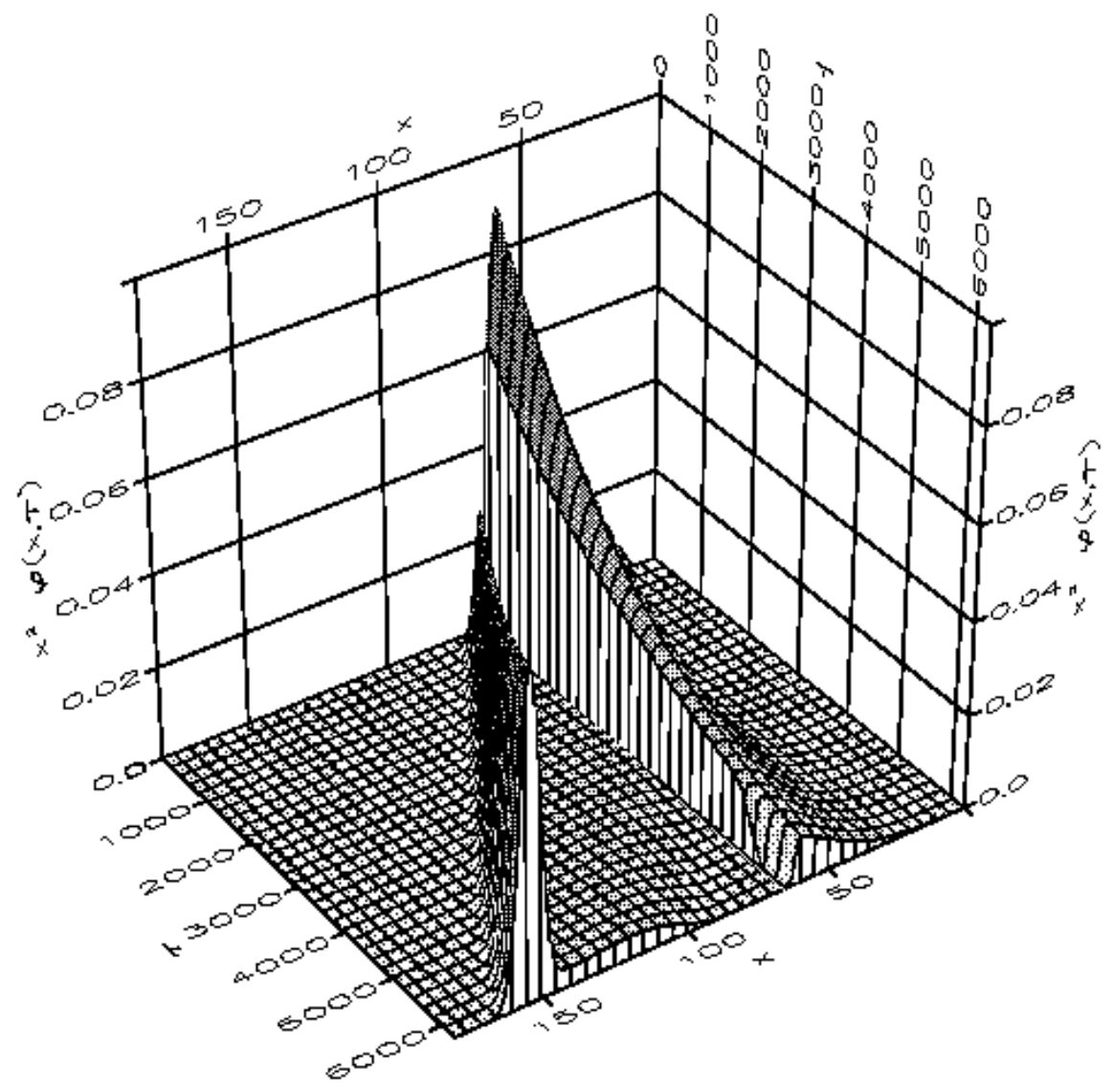




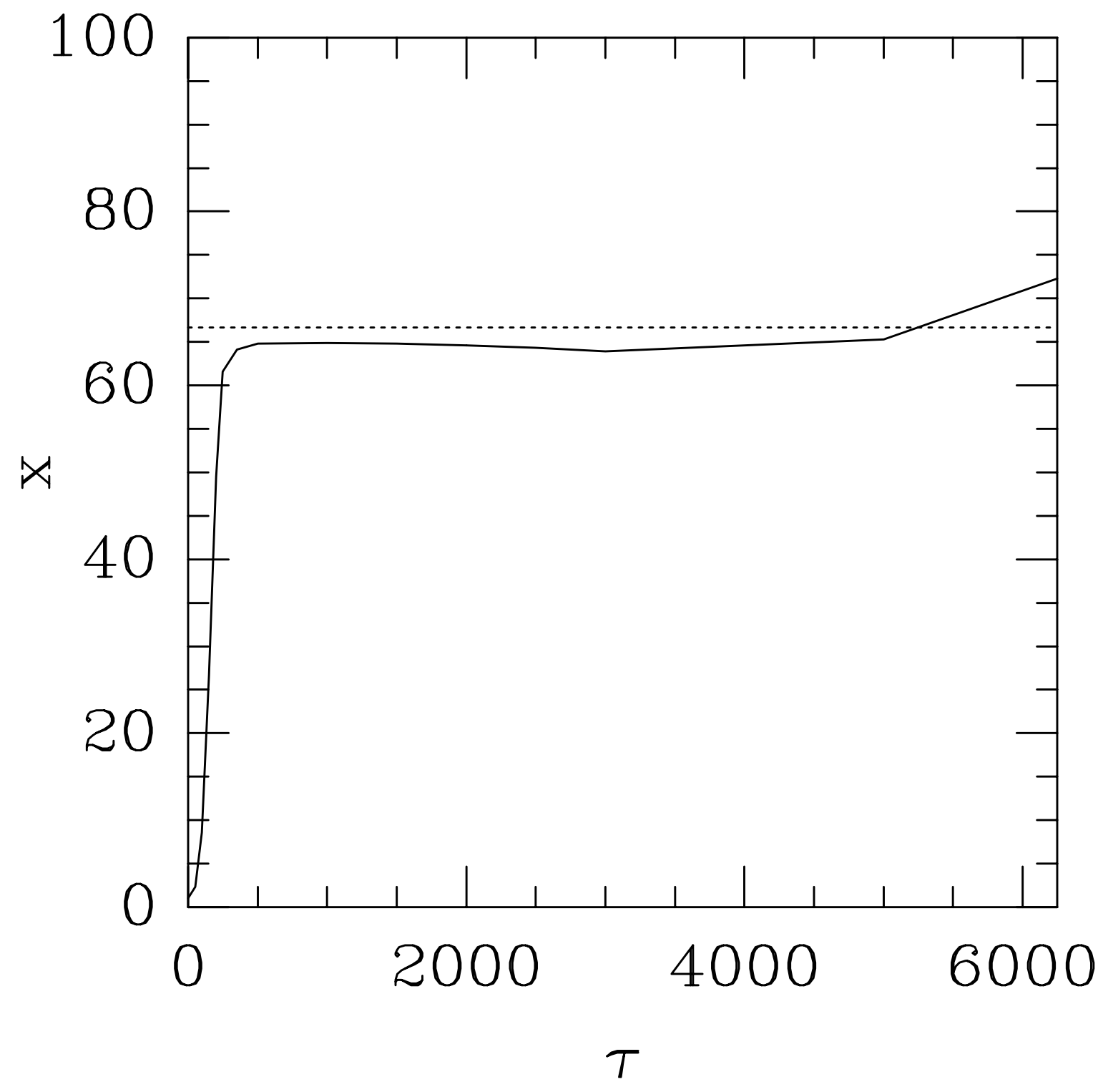

